\title{
Application-layer Performance Analysis of PRIME in Smart Metering Networks
}

\author{
Julio A. Corchado*, Eduardo Manero ${ }^{\dagger}$, José Antonio Cortés*, Alfredo Sanz ${ }^{\dagger}$, Luis Díez* \\ *Dpto. de Ingeniería de Comunicaciones \\ E.T.S.I. de Telecomunicación, University of Málaga \\ Campus de Teatinos s/n, 29071 Málaga (Spain) \\ $\dagger$ Atmel Spain \\ Torre C2, Polígono Puerta Norte, A-23, 50820 Zaragoza (Spain)
}

\begin{abstract}
This paper assesses the performance of actual PRIME v1.3.6 and PRIME v1.4 systems when used for Smart Metering applications. The analysis is performed at the application level using the DLMS/COSEM stack. Hence, it considers performance indicators that are of practical interest for distribution system operators, such as the availability and the average time needed to read the energy load profile of all the meters.

To this end, two test networks with 112 smart meters have been deployed in the laboratory (to ensure the stability of the network). In one of them all the Service Nodes communicate directly with the Base Node, while there exist up to 5 switching levels in the other tested network.

First, the PRIME v1.3.6 system is evaluated, stressing the significant performance gain that can be obtained by implementing some MAC layer strategies, which are compatible with the specification but not specifically defined on it. Then, the improvement offered by the PRIME v1.4 system is assessed.
\end{abstract}

\section{INTRODUCTION}

Smart Metering, also referred to as AMR (Automatic Meter Reading), can be loosely defined as the application that allows the remote consumption reading and the diagnosis and basic operation of the meters. The communication network and the management elements that support it are usually referred to as AMI (Advanced Metering Infrastructure). Its deployment is one of the Smart Grid parts that is gathering more operational and research efforts [1]. To this end, high data rate NB-PLC (Narrowband Power Line Communications) systems like the ones promoted by the PRIME and G3-PLC industrial alliances, or the one defined in the IEEE P1901.2 [2], seem to be one of the most suitable solutions for this purpose [3].

The first version of the PRIME (PoweRline Intelligent Metering Evolution) specification, referred to as PRIME v1.3.6 [4], has been standardized by the ITU-T in the G.9904 Recommendation [5]. It defines the PHY (Physical), MAC (Medium Access Control) and convergence layers, along with the management plane, of a system that works in the CENELECA band $(35-91 \mathrm{kHz})$. A new version of the PRIME system, denoted as PRIME v1.4, has been recently released [6]. It incorporates some enhancements both at the physical and MAC layers, along with a bandwidth extension up to $500 \mathrm{kHz}$, and ensures full backward compatibility with PRIME v1.3.6 devices.

PRIME v1.3.6 is extensively employed in Smart Metering networks, where DLMS/COSEM (Device Language Message
Specification/Companion Specification for Energy Metering) is used at the application layer [7], [8]. Interesting performance indicators at this level are the cycle time (i.e. the time needed to read all the meters managed by a Base Node) and the availability rate of each meter (i.e., the rate of successful meter reading attempts). Despite the importance of these indicators for the DSO (Distribution System Operator), most of the studies accomplished up to now have focused on the analysis of the physical layer performance [9], [10], [11], [12]. Hence, the number of works assessing the performance at the application level is much lower and are either obtained by means of simulations [13], [14] or focused in the deployment aspects and the management tools used by the DSO [15], [16], [17]. Due to the relative novelty of the PRIME v1.4 specification, which was released in October 2014, there are almost no works in the literature dealing with this system.

In this context, we make two main contributions:

- We assess the performance of PRIME v1.3.6 in Smart Metering networks. The accomplished analysis highlights the importance of an adequate MAC layer parameterization and the potential of this layer to improve the overall system performance. The latter is illustrated by evaluating the performance gain obtained by adding some MAC layer strategies, compatible with the specification but not specifically defined on it.

- We compare the performance of PRIME v1.4 with respect to PRIME v1.3.6 when used in Smart Metering networks.

Both studies are accomplished using actual PRIME devices and performance is measured at the application layer. Since results are topology-dependent, two extreme networks are considered: one in which all Service Nodes can directly connect to the Base Node and another with up to 5 switching levels.

The rest of the paper is organized as follows. A brief review of the PRIME specifications is given in Section II. Section III describes the network configurations used in the study. The results of the performance analysis are presented and discussed in Section IV. Finally, Section V summarizes the main aspects of the paper. 


\section{REVIEW OF THE PRIME SPECIFICATIONS}

This brief review focuses on the aspects that are further addressed in the performance analysis.

\section{A. System architecture}

The system architecture of both PRIME specifications is shown in Fig. 1. As seen, they define the PHY layer, the MAC layer and the convergence layer, as well as the management plane. The MAC layer performs key functions like medium access control, resource allocation, connection management and topology resolution.

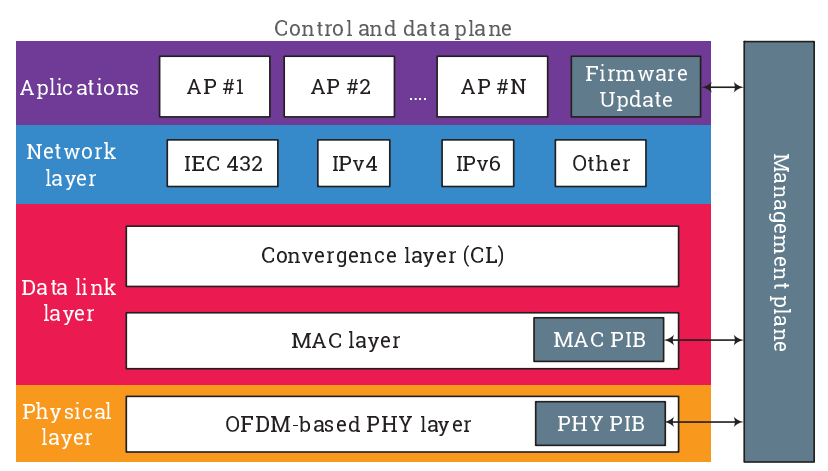

Figure 1. PRIME System architecture.

PRIME networks consist of a set of subnetworks deployed in the context of a transformer station. Subnetworks have a tree-like topology and are comprised of a Base Node (the root) and multiple Service Nodes (either branch or leaves). The Base Node manages the subnetwork. It transmits the beacons $(\mathrm{BCN})$ used for network synchronization and performs key management functions like Service Nodes registration, connection setup and management, resource allocation and encryption, among others.

In Smart Metering applications, the Data Concentrator is the network element that runs the DLSM/COSEM protocol. It is responsible for polling the smart meters connected to the subnetwork and for sending the retrieved data to the management system. It may be implemented as an independent device that connects to the Base Node by means of the "DLMS over TCP" functionality defined by the PRIME Alliance or, alternatively, bundled with the Base Node in a single device.

Service Nodes have two responsibilities: connecting to the subnetwork to send their own information and, if needed, relaying the information from neighbor Service Nodes. When a Service Node transmits only its own information, it is referred to as Terminal, while it is denoted as Switch when it also relays information from neighbor nodes. Node promotion (from Terminal to Switch) and demotion (from Switch to Terminal) are dynamically managed by the Base Node.

\section{B. MAC layer}

The MAC layer uses a proactive routing algorithm. Hence, network nodes react to topology changes, even if no traffic is to be sent at the moment. This causes a routing traffic overhead but minimizes the delay when new data has to be sent. To this end, the Keep-Alive process is employed. It uses the ALV control packets, which are periodically exchanged between a Service Node, the Service Node above it (if any) and the Base Node. Upon the reception of an ALV message, the Service Node has to send an ALV response before the keep alive time configured in the parameter ALV.TIME has passed. Otherwise, the Base Node will consider this node as unregistered. This parameter strongly influences the cycle time in Smart Metering applications. A low ALV.TIME allows fast network topology changes, reducing the delay of the next smart meters read process, but also augments the overhead traffic, which increases the number of collisions and, in turn, the cycle time.

\section{Physical layer}

Both PRIME versions define an OFDM (Orthogonal Frequency Division Multiplexing)-based physical layer. Differential (in frequency) modulations are employed in both cases. PRIME v1.3.6 employs DBPSK, DQPSK and D8PSK, which can be used with or without a rate $1 / 2$ convolutional code with a constraint length of 7. PRIME v1.4 defines two additional modes denoted as DBPSK robust and DQPSK robust which repeat each convolutionally encoded OFDM symbol by 4 . The preamble and the header of the PHY layer frames used in the robust modes are also four times larger than the normal ones.

\section{DESCRIPTION OF THE CONSIDERED SCENARIOS}

Networks employed in the performance assessment have been deployed in the premises of Atmel Spain, using the structure shown in 2 . The laboratory setup ensures the network stability needed to perform repeatable performance measurements.

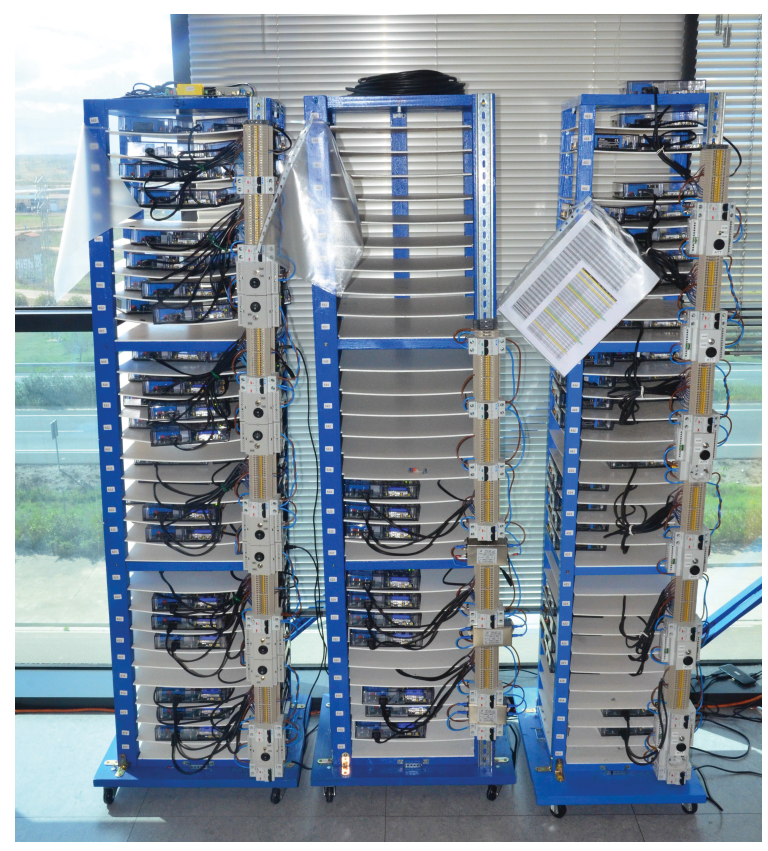

Figure 2. Set-up used to deploy the tested networks. 
Two networks topologies consisting of the Base Node and 112 smart meters have been deployed. In one of them all the meters have direct connection to the Base Node. This will be referred to as flat network. In the other, a set of attenuators are employed to create multiple levels of switching, as shown in Fig. 3. This set-up will be referred to as multilevel network. The employed attenuation values are intended to create around 5 levels of switching. Ideally, among all the Service Nodes experiencing the same attenuation level, only one of them should act as switch. However, in practice, it may happen that two Service Nodes with the same attenuation level act as switches. It may also happen that there are no switches at the $i$ th level because Service Nodes located in the $(i+1)$ th level can directly communicate with one switch located in the $(i-1)$ th level.

The employed Service Nodes are equipped with the Atmel SAM4CP16B development kit, which integrates the ATPL230A modem. It implements both a PRIME v1.3.6. and PRIME v1.4 system. When assessing the performance of PRIME v1.3.6, two Base Nodes have been tested in each network topology: one is developed by Atmel and the other is embedded in the employed data concentrator, which is manufactured by another vendor. The Atmel Base Node consists of a SAME70Q21 microcontroller and an ATPL230 modem. It connects to the data concentrator by means of the "DLMS over TCP" feature defined by the PRIME Alliance. The performance of PRIME v1.4 has been evaluated only in the multilevel network topology and exclusively uses the Atmel Base Node because currently there are no commercially available Base Nodes from other manufacturers.

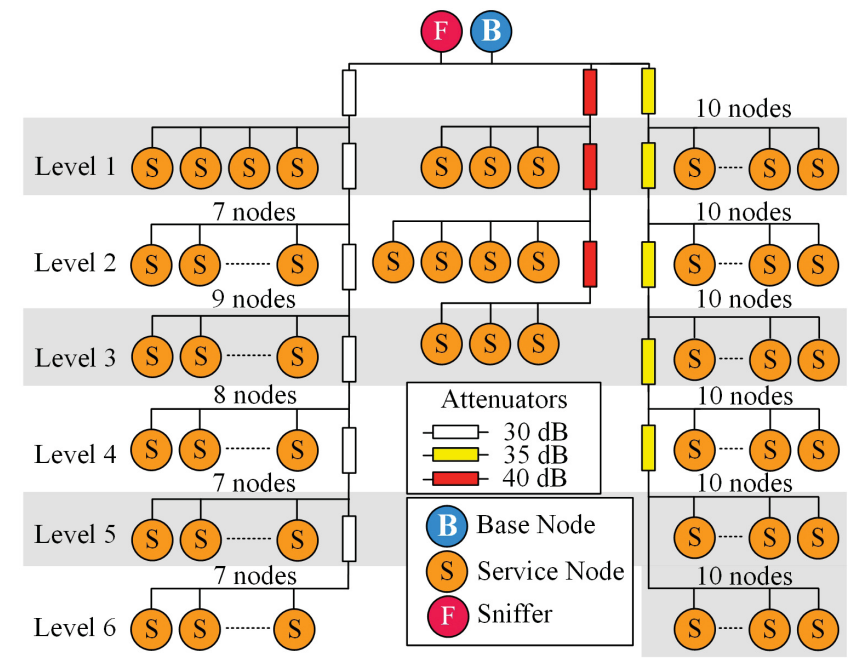

Figure 3. Multilevel network topology.

Frames transmitted and received by the Base Node are captured by a packet sniffer, developed by Atmel Spain, bundled with the SAM4CP16B development kit. This information is processed offline to calculate the desired performance values. To this end, only frames whose origin and destination are the Base Node are taken into account, i.e. frames relayed by first level switches to Service Nodes located below it but that also received at the Base Node are not considered.

\section{Performance ANAlysis}

The interesting performance indicators at the application layer (DLMS/COSEM) are the availability rate of each meter and the cycle time. The former is defined as the rate of successful meter reading attempts (number of successful meter reads/number of meter read attempts). The latter is the time needed to read the COSEM objects of all the meters. As indicated in Section III, two Base Nodes manufactured by different vendors are tested when PRIME v1.3.6 is assessed. The objective is to stress the decisive influence of the MAC layer implementation of the Base Node in the network performance. The evaluation of PRIME v1.4 is done using exclusively the Atmel Base Node and employing channel 1, to allow a fair comparison with PRIME v1.3.6.

\section{A. PRIME v1.3.6}

Table I shows the performance values obtained in the flat network. As seen, both Base Nodes yield the same availability rates. On the contrary, both the mean and the standard deviation of the cycle time are much lower when the Atmel Base Node is employed. Thus, the average cycle time is about $47.4 \%$ lower than the one obtained with the Base Node from the other manufacturer.

Table I

Performance VAlues IN THE Flat NETWORK

\begin{tabular}{ccc}
$\begin{array}{c}\text { Base Node } \\
\text { vendor }\end{array}$ & $\begin{array}{c}\text { Availability rate }(\%) \\
\text { [Number of meters with } \\
\text { the given availability rate] }\end{array}$ & $\begin{array}{c}\text { Average cycle time } \\
\text { (minutes:seconds) } \\
\text { [Standard deviation of } \\
\text { the cycle time (seconds)] }\end{array}$ \\
\hline Atmel & 100 & $3: 54$ \\
& {$[112]$} & {$[9]$} \\
Other & 100 & $7: 25$ \\
& {$[112]$} & {$[32]$} \\
\hline
\end{tabular}

There are two causes for the significant performance differences shown in Table I, and both are due to the MAC layer of the Base Node. One of the reasons is that the default values given by Atmel to some MAC parameters happen to be more appropriate for the considered scenario. The other is the use of some strategies that reduce the number of collisions. These Atmel-proprietary strategies are compatible with the PRIME specification but are not defined on it.

The first cause of the enhanced performance achieved by the Atmel Base Node is the value of the keep alive time that it employs. By default, Atmel fixes ALV.TIME to 512 seconds, while the other manufacturer fixes it to 256 seconds. The effect of the different keep alive times can be clearly observed in Fig. 4(a), where the number of ALV frames transmitted (downlink) and received (uplink) by the Base Node in the flat network topology in each cycle is depicted. As seen, the use of the Atmel Base Node results in a much lower number of ALV frames. In addition, the number of uplink and downlink ALV frames is almost equal, while the number 
of downlink frames is higher than the number of uplink ones when the Base Node from the other vendor is employed. The reason is that some of the ALV frames collide and, since they are not correctly received, must be retransmited. Both effects increase the traffic volume, as shown in Fig. 4(b) and Fig. 4(c). The latter shows the overall number of uplink and downlink frames in each cycle, while in the former the BCN and ALV frames are not counted. As seen, in both cases the number of frames transmitted by the Atmel Base Node is lower than the amount of frames transmitted by the Base Node from the other manufacturer, which results in a much shorter cycle time. It is interesting to note that the the number of uplink frames in Fig. 4(b) is larger than the number of downlink ones. This is in accordance with the fact that the minimum number of uplink and downlink data frames needed to read each meter is 14 and 9, respectively, when piggibacking is used and 20 and 14 when acknowledgments are transmitted using ACK frames.
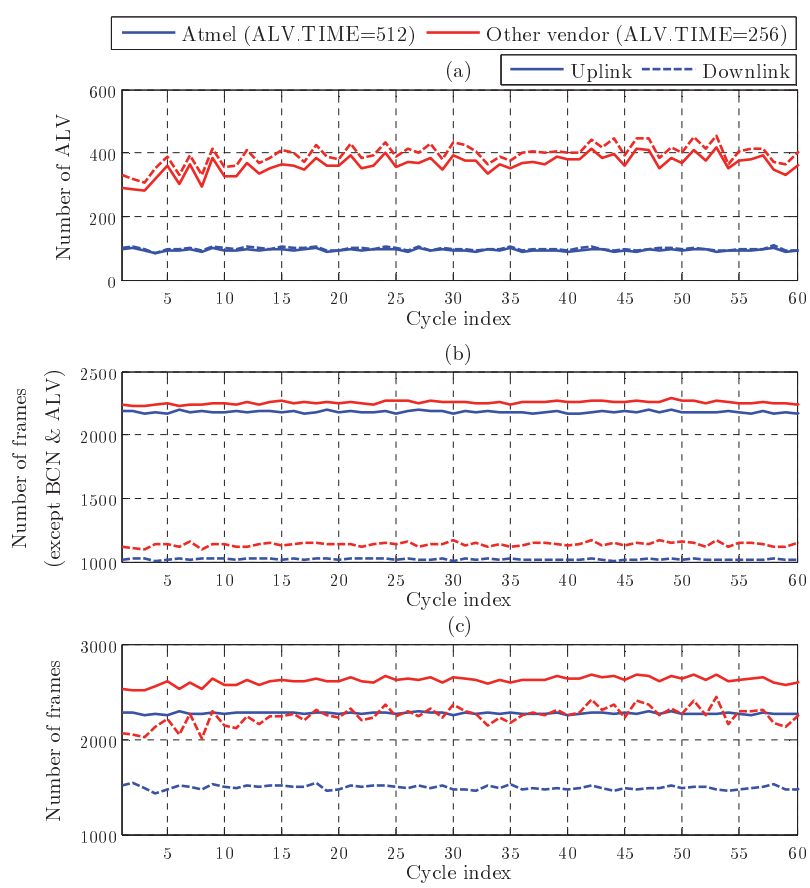

Figure 4. Number of uplink and downlink frames in the flat network using PRIME v1.3.6: (a) ALV frames (b) all types of frames except BCN and ALV, (c) all types of frames.

The most appropriate value for ALV.TIME depends on the rate at which network changes (significant enough to cause link outages) occur. Since the considered network is static, the larger value of ALV.TIME in the Atmel Base Node is partially responsible for its improved performance. However, ALV frames represent only about $22.5 \%$ of the overall number of frames (excluding BCN and ALV) when the Base Node from the other vendor is employed, as observed by comparing Fig. 4(a) and Fig. 4(b). This suggests that performance differences shown in Table I should be also due to the Atmel-proprietary MAC strategies that reduce the number of collisions.
To assess the effectiveness of these techniques, the performance in the multilevel network topology is evaluated with $A L V . T I M E=256$ in both Base Nodes. Table II summarizes the obtained results. As expected, performance values are worse than in the flat network. However, the Atmel Base Node still performs significantly better than the one of the other vendor. As seen, all meters were successfully read in all cycles when the Atmel Base Node is used. On the contrary, when the Base Node from the other vendor is employed, there are five meters (out of the 112) with availability rates lower than $100 \%$. Performance differences are particularly significant in the average cycle time, where the Atmel Base Node yields a value $38.9 \%$ lower than the one of the other manufacturer.

Table II

Performance VAlues in the MUltileVel Network

\begin{tabular}{ccc}
$\begin{array}{c}\text { Base Node } \\
\text { vendor }\end{array}$ & $\begin{array}{c}\text { Availability rate }(\%) \\
\text { [Number of meters with } \\
\text { the given availability rate] }\end{array}$ & $\begin{array}{c}\text { Average cycle time } \\
\text { (minutes:seconds) } \\
\text { [Standard deviation of } \\
\text { the cycle time (seconds)] }\end{array}$ \\
\hline Atmel & 100 & $13: 07$ \\
& {$[112]$} & {$[9]$} \\
& 97.5 & \\
& {$[1]$} & $21: 29$ \\
Other & 98.4 & {$[46]$} \\
& {$[4]$} & \\
& 100 & \\
\end{tabular}

To illustrate the reason for the performance differences shown in Table II, Fig. 5(a) shows the number of ALV frames exchanged in each cycle. As seen, the number of uplink and downlink ALV frames is almost equal when the Atmel Base Node is used (curves are indistinguishable). On the contrary, about $17 \%$ of the downlink ALV frames sent by the Base Node from the other vendor are lost due to collisions. The time needed to resolve these collisions results in longer cycle times, which in turn causes the number of uplink ALV frames to be larger than when the Atmel Base Node is employed.

Fig. 5(b) depicts the number of uplink and downlink frames (excluding BCN and ALV ones) exchanged by the Base Node. These values are quite similar with both Base Nodes. However, as shown in Fig. 5(c), the overall number of downlink frames sent by the Base Node of the other manufacturer is much larger than the one of the Atmel Base Node (around 5000 frames per cycle vs 4000 frames per cycle).

The lower number of frames exchanged in each cycle when the Atmel Base Node is employed is due to the collision avoidance strategies that it implements. One of these strategies makes the Base Node to take into account the type of frames that have been just transmitted before transmitting a new one. This prevents situations like the one shown in Fig. 6, which shows a sniffer register acquired in the multilevel network when the Base Node from the other manufacturer is employed. As seen, the Base Node transmits a data frame with idFrame 30295. It has to be answered by the Service Node but, before receiving the awaited answer, the Base Node transmits two 
ALV frames headed for two different Service Nodes. One with idFrame 30299, which is further relayed by a switch located at the first level (idFrame 30300), an another one with idFrame 30303. This yields a situation were potential collisions between the response to the data and to the ALV frames may occur. Indeed, while the answer to the data frame sent by the Base Node is received in frames with idFrames 30305 to 30307 , the responses to the ALV frames sent by the corresponding Service Nodes are never received at the Base Node.
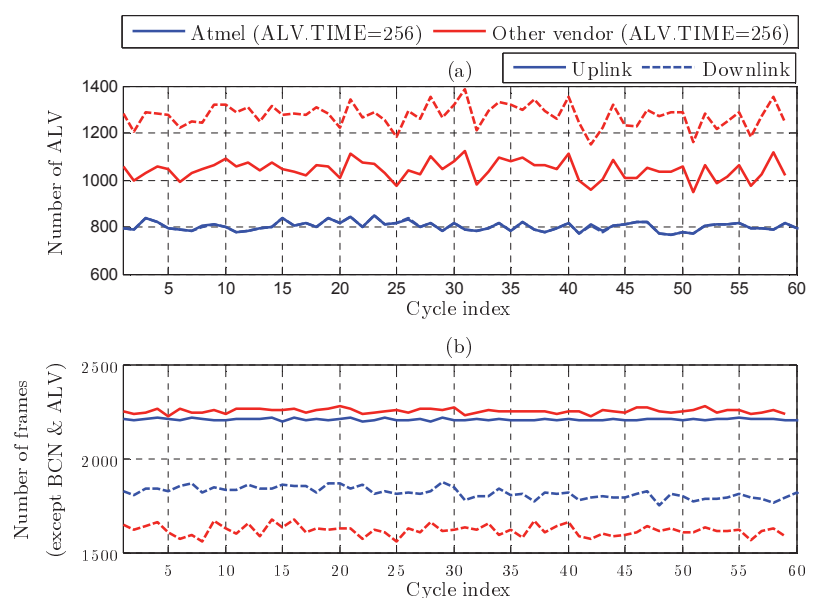

(c)

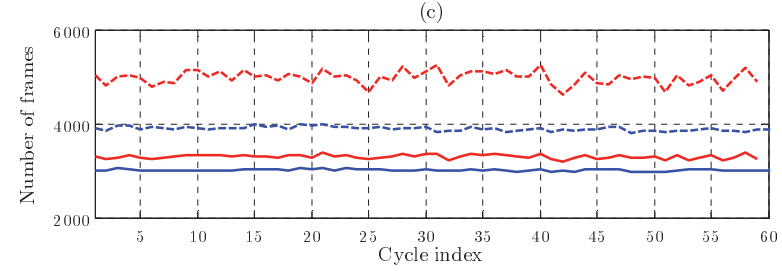

Figure 5. Number of uplink and downlink frames in the multilevel network using PRIME v1.3.6: (a) ALV frames, (b) all types of frames except BCN and ALV, (c) all types of frames.

\begin{tabular}{|c|c|c|c|c|c|c|c|}
\hline idframe & TimeStamp & Modulation & Duration & Up_Down & Level & PduType & GenType \\
\hline 30295 & 2016-02-08 11:42:53.565 & DBPSK_C & 15,48 & Down & 0 & GEN & DAT \\
\hline 30296 & $2016-02-08$ 11:42:53.608 & DBPSK_C & 11 & Down & 0 & $\mathrm{BCN}$ & $\cdots$ \\
\hline 30297 & 2016-02-08 11:42:53.638 & DBPSK_C & 11 & Down & 1 & $\mathrm{BCN}$ & $\cdots$ \\
\hline 30298 & 2016-02-08 11:42:53.687 & DBPSK_C & 15,48 & Down & 1 & GEN & DAT \\
\hline 30299 & 2016-02-08 11:42:53.876 & DBPSK_C & 11 & Down & 0 & GEN & ALV \\
\hline 30300 & 2016-02-08 11:42:53.915 & DBPSK_C & 11 & Down & 1 & GEN & ALV \\
\hline 30301 & 2016-02-08 11:42:54.228 & DBPSK_C & 11 & Down & 0 & $\mathrm{BCN}$ & $\cdots$ \\
\hline 30302 & 2016-02-08 11:42:54.262 & DBPSK_C & 11 & Down & 1 & $\mathrm{BCN}$ & $\cdots$ \\
\hline 30303 & 2016-02-08 11:42:54.338 & DBPSK_C & 11 & Down & 0 & GEN & ALV \\
\hline 30304 & 2016-02-08 11:42:54.373 & DBPSK_C & 11 & Down & 1 & GEN & ALV \\
\hline 30305 & 2016-02-08 11:42:54.442 & DBPSK_C & 31,16 & Up & 0 & GEN & DAT \\
\hline 30306 & 2016-02-08 11:42:54.494 & DBPSK_C & 31,16 & Up & 0 & GEN & DAT \\
\hline 30307 & 2016-02-08 11:42:54.544 & DBPSK_C & 31,16 & Up & 0 & GEN & DAT \\
\hline
\end{tabular}

Figure 6. Sniffer register illustrating a potential collision situation that occurs when the Base Node from the other manufacturer is employed in the multilevel network using PRIME v1.3.6.

\section{B. PRIME v1.4}

The objective of this subsection is to illustrate the performance gain provided by PRIME v1.4 with respect to PRIME v1.3.6. To this end, only the worst case scenario, i.e. the multilevel network topology is assessed. Since currently there are no commercially available PRIME v1.4 Base Nodes from other manufacturers, only the Atmel one is used.

In these circumstances, the average cycle time falls from 13:07 (obtained with PRIME v1.3.6) to 9:27 (28\% reduction), while the standard deviation remains almost equal (10 seconds). The cause for the cycle time shortening is twofold. One reason is the reduced number of ALV frames, whose functionality is now embedded in the header of the data frames. Hence, ALV frames are only transmitted during the first cycle. This can be clearly observed in Fig. 7(a), where the number of ALV frames transmitted and received by the Base Node in the first 10 cycles is shown. As seen, there are no ALV frames from the second cycle on. This slightly reduces the traffic volume in the network, as illustrated in Fig. 7(b).
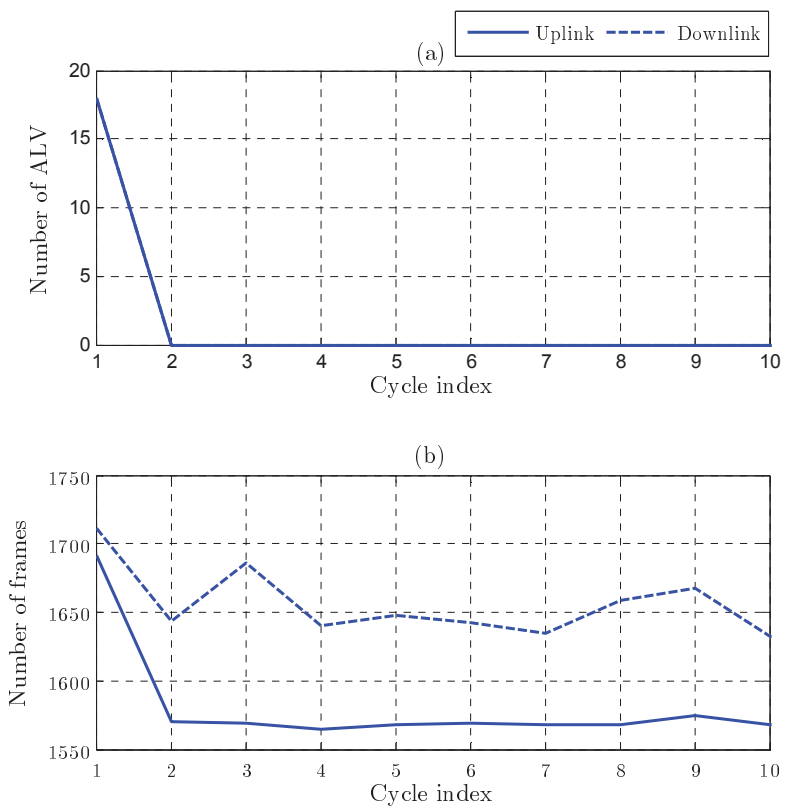

Figure 7. Number of uplink and downlink frames in the multilevel network using PRIME v1.4: (a) ALV frames (b) all types of frames.

The second reason for the shorter cycle times is the reduction in the number of frames that occurs because of the enhanced mechanism used to change the employed modulation and coding scheme. In PRIME v1.3.6 this is done by means of specific asynchronous messages exchanged between the terminal and the switch, which cause multiple collisions due to the hidden node problem. However, in PRIME v1.4 the link quality information used for this purpose is embedded in the header of the generic data frames. This is crearly illustrated in Fig. 8, where the frame with idFrame 5954 is sent by the Base Node to a given Service Node using the DBPSK robust mode. The Service Node response (idFrame 5955) also uses this mode. However, the next frames exchanged by the Base 
Node and this Service Node (idFrame 5957 and 5958) are sent using DBPSK with convolutional coding. The spectral efficiency of the transmissions is continuously increased up to idFrame 5967, in which the Base Node uses D8PSK. As shown in Fig. 8, this process results in a significant reduction of the duration of the frames exchanged between the Base Node and the Service Node.

As a result, the overall number of frames in the PRIME v1.4 network is significantly lower than in the PRIME v1.3.6 one, as can be observed by comparing Fig. 7(b) and Fig. 5(c).

\begin{tabular}{|c|c|c|c|c|c|c|c|}
\hline idframe & TimeStamp & Modulation & Duration & Up_Down & Level & PduType & GenType \\
\hline 5954 & $2016-04-21$ 17:09:58.307 & R_DBPSK & 106,75 & Down & 0 & GEN14 & DAT \\
\hline 5955 & 2016-04-21 17:09:58.383 & R_DBPSK & 44,03 & Up & 0 & GEN14 & DAT \\
\hline 5956 & 2016-04-21 17:09:58.475 & DBPSK_C & 24,44 & Down & 0 & GEN14 & DAT \\
\hline 5957 & 2016-04-21 17:09:58.549 & DBPSK_C & 28,92 & Up & 0 & GEN14 & DAT \\
\hline 5958 & 2016-04-21 17:09:58.672 & DBPSK_C & 17,72 & Down & 0 & GEN14 & DAT \\
\hline 5959 & 2016-04-21 17:09:58.735 & DQPSK_C & 13,24 & Up & 0 & GEN14 & DAT \\
\hline 5960 & 2016-04-21 17:09:58.867 & R_DBPSK & 52,99 & Down & 0 & BCN14 & --- \\
\hline 5961 & $2016-04-21$ 17:09:58.961 & D8PSK_C & 13,24 & Down & 0 & GEN14 & DAT \\
\hline 5962 & 2016-04-21 17:09:59.029 & D8PSK_C & 13,24 & Down & 0 & GEN14 & DAT \\
\hline 5963 & 2016-04-21 17:09:59.074 & D8PSK_C & 17,72 & Up & 0 & GEN14 & DAT \\
\hline 5964 & 2016-04-21 17:09:59.115 & D8PSK_C & 17,72 & Up & 0 & GEN14 & DAT \\
\hline 5965 & 2016-04-21 17:09:59.165 & D8PSK_C & 17,72 & Up & 0 & GEN14 & DAT \\
\hline 5966 & 2016-04-21 17:09:59.213 & D8PSK_C & 17,72 & Up & 0 & GEN14 & DAT \\
\hline 5967 & $2016-04-21$ 17:09:59.348 & D8PSK & 8,76 & Down & 0 & GEN14 & DAT \\
\hline
\end{tabular}

Figure 8. Sniffer register illustrating that, when using PRIME v1.4, modulation and coding scheme changes are driven by the link quality information embedded in the header of the generic data frames.

\section{CONCLUSION}

This paper has assessed the performance of PRIME v1.3.6 and PRIME v1.4 in Smart Metering networks. Since performance is topology-dependent, two extreme networks have been considered. A best case one in which all Service Nodes can directly connect to the Base Node, and a worst case network in which multiple switching levels are created using attenuators. Both networks consist in 112 actual devices deployed in a laboratory environment to ensure the repeatability of the measurements. The performance has been evaluated at the application-level layer (DLMS/COSEM).

The analysis of PRIME v1.3.6 has stressed the potential of the MAC layer to improve the overall system performance. Hence, an average cycle time reduction of $38.9 \%$, with respect to the value achieved by the Base Node manufactured by another vendor, has been obtained in the multilevel network by using an Atmel Base Node that incorporates a set of proprietary strategies at the MAC layer. These strategies are compatible with the specification but are not specifically defined on it. The comparison of the performance achieved by these two Base Nodes has also highlighted the importance of an adequate selection of the keep alive time configured at the MAC layer.

Finally, it has been shown that the use of PRIME v1.4 in the worst case network yields an average cycle time reduction of $28 \%$ with respect to PRIME v1.3.6. The main sources of this improvement are the embedding of the keep alive functionality and the information used to switch the employed modulation and coding scheme in the header of the generic data packets. Both elements lower the number of collisions by reducing the traffic volume and, in the latter case, also by shortening the frame duration.

\section{ACKNOWLEDGMENT}

The authors thank Atmel corporation for the support provided to accomplish this work.

\section{REFERENCES}

[1] A. Sendin, I. Peña, and P. Angueira, "Strategies for power line communications smart metering network deployment," Energies, vol. 2014, no. 7 , pp. 2377-2420, 2014.

[2] IEEE P1901.2, "IEEE standard for low-frequency (less than $500 \mathrm{kHz}$ ) narrowband power line communications for Smart Grid applications," October 2013.

[3] S. Galli, A. Scaglione, and Z. Wang, "For the grid and through the grid: The role of power line communications in the Smart Grid," Proceedings of the IEEE, vol. 99, no. 6, pp. 998-1027, June 2011.

[4] PRIME Alliance TWG, "Draft Specification for PoweRline Intelligent Metering Evolution," Release 1.3.6.

[5] "Narrowband orthogonal frequency division multiplexing power line communication transceivers for PRIME networks," ITU-T Recommendation G.9904, October 2012

[6] PRIME Alliance TWG, "Specification for PoweRline Intelligent Metering Evolution," 2014, Release 1.4.0.

[7] DLMS UA 1000-2, "Green Book: Architecture and Protocols," 2014, Ed.8.0.

[8] DLMS UA 1000-1, "Blue book: COSEM Identification system and Interface Objects," 2014, Ed.12.0.

[9] M. Hoch, "Comparison of PLC G3 and PRIME," in Proceedings of the IEEE International Symposium on Power Line Communications and its Applications (ISPLC), March 2011, pp. 165-169.

[10] M. Korki, C. Zhang, and H. L. Vu, "Performance Evaluation of PRIME in Smart Grid," in Proceedings IEEE International Conference on Smart Grid Communications, 2013.

[11] J. Matanza, S. Alexandres, and C. Rodríguez-Morcillo, "Performance evaluation of two narrowband PLC systems: PRIME and G3," Computer Standards \& Interfaces, vol. 36, p. 198208, 2013.

[12] M. Wolkerstorfer, B. Schweighofer, H. Wegleiter, D. Statovci, H. Schwaiger, and W. Lackner, "Measurement and simulation framework for throughput evaluation of narrowband power line communication links in low-voltage grids," Journal of Network and Computer Applications, vol. 59, pp. 285-300, 2016.

[13] A. Sanz, P. J. Piñero, D. Montoro, and J. I. García, "High-accuracy Distributed Simulation Environment for PRIME Networks Analysis and Improvement," in Proceedings of the IEEE International Symposium on Power Line Communications and its Applications (ISPLC), March 2012.

[14] J. Matanza, S. Alexandres, and C. Rodríguez-Morcillo, "Advanced metering infrastructure performance using European low-voltage power line communication networks," IET Communications, vol. 8, no. 7, pp. 1041-1047, 2014

[15] I. Berganza, A. Sendin, A. Arzuaga, M. Sharma, and B. Varadarajan, "PRIME on-field deployment," in Proceedings of the IEEE International Conference on Smart Grid Communications, 2011

[16] A. Sendin, I. Berganza, A. Arzuaga, A. Pulkkinen, and I. Han Kim, "Performance results from 100,000+ PRIME smart meters deployment in Spain," in Proceedings of the IEEE International Conference on Smart Grid Communications (SmartGridComm), November 2012, pp. $145-150$.

[17] A. Fernandez, A. Sendin, I. Urrutia, J. Mateo, P. Angueira, and J. Ferro, "Analysis of PRIME PLC Smart Metering Networks Performance," in International Conference on Renewable Energies and Power Quality, 2013. 